\title{
Websites Designed for Students with Special Needs
}

\author{
Abrar Naif Alanazi (first and Corresponding author) \\ Parent organizations: ministry of education \\ Postal code: 11432 \\ P.o. box: 5701 \\ Phone: 00966543236404 \\ Fax: 00966112590555 \\ Website: www. Imamu.edu.sa \\ E-mail: aalanazi@imamu.edu.sa \\ Abrar.naif@hotmail.com
}

\begin{abstract}
According to Friedman \& Bryen, (2007), a person with a disability is one with a long-term physical, intellectual, mental or sensory impairment, which interferes with their social equality in relation to others. Disability, thus, varies widely across countries. Borg, Berman-Bieler, \& Khasnabis (2015), indicated that by 2004, about 93 million children aged 14-years and below, suffered from moderate to severe disabilities. Examples of common disabilities include hearing loss, down syndrome, cerebral palsy, brain injury, intellectual and learning disability, congenital anomalies, muscular dystrophy, blindness, spinal code injuries, visual loss and speech impairments (Borg, Berman-Bieler, \& Khasnabis, 2015)

Assistive technology has become a common term in discussions regarding children with disabilities. This term includes technology, which assists disabled persons to carry out their activities or to facilitate their activities. They include technologies used to facilitate mobility, vision, hearing, communication and cognition. This paper will explore how web designs can be used to assist in the learning process. One such common disability is that of dyslexia, which is a medical condition that makes it difficult for students to read and learn and which lends itself well to assistive technologies (HKSAR, 2008).

It is estimated that one in every 10 people have dyslexia (HKSAR, 2008). The condition exists across a range of cultures, abilities and conditions and is usually gene related and thus, runs in the family. While it cannot be cured, there are practical approaches that can help overcome the barriers presented by the condition. Dyslexia can be termed as a learning difference meaning that the brain approaches tasks differently to other people and affects the way people communicate (HKSAR, 2008). Since dyslexia does not affect the individual's intelligence, it can be managed by using a different communication model in the learning process (Friedman \& Bryen, 2007). Assistive technology such as the use of specialized websites can, therefore, be used to assist children with dyslexia.
\end{abstract}

Keywords: Dyslexia, Cognitive Disability, Technology, Accessibility, and Autism.

DOI: $10.7176 / \mathrm{JEP} / 10-30-14$

Publication date:October $31^{\text {st }} 2019$

\section{Abbreviations \\ 1. WWW - World Wide Web. \\ 2. NIDRR - National Institute on Disability Research and Rehabilitation \\ 3. WHO - World Health Organization \\ Introduction}

This research explores web accessibility for people with cognitive disabilities. It will focus on web accessibility and the guidelines to be followed when designing web accessibility for children with cognitive disabilities (HKSAR, 2008). Cognitive disabilities include conditions such as aphasia, dyslexia, mental retardation, autism and learning disabilities. These conditions pose numerous accessibility barriers to internet use by those who suffer from such conditions. The difficulties experienced by sufferers include memory problems, conceptualization, perception, attention and problem-solving disabilities (HKSAR, 2008). These difficulties, therefore, result in a cognitive disability related to the proper use of the internet due to limitations in terms of reading comprehension, slow learning, complexity in the learning process, lowered visual acuity, lower information threshold and the difficulty in identifying images, mouse control, comprehending the screen, tracing and clicking on small images and letters in drop down boxes and icons (Dobransky \& Hargittai, 2016). 
Literature review. In the past few years, various steps have been taken to address web accessibility for all types of web users by persons with visual, physical and sensory disabilities. However, much still needs to be done. Accessibility to the internet by all kinds of people has been a consideration since the beginning of the World Wide Web (WWW). The introduction of the WWW by Tim Berners-Lee was aimed at removing the barriers associated with web accessibility by people with various disabilities (Borg, Berman-Bieler, \& Khasnabis, 2015).

Screen readers were developed in early 1980s for visually impaired people to enable them to work on computers, which were later modified to enable internet access through incorporated text to speech functionalities (Borg, Berman-Bieler, \& Khasnabis, 2015). The text-to-speech has replaced the text tags and enlarged text zoom features, previously used. Further, web browsers have been developed as assistive technology on an ongoing basis. The 1999 World Wide Web Accessibility Initiative provided guidelines on web content accessibility, which include functionalities such as web pages that should legitimately display a coveted Bobby symbol, incorporating guidelines and compliance as a top priority item. Screen readers have been found to be complex, therefore, more research is being conducted in this area. To address web accessibility for internet users with cognitive disabilities, the following literature analysis was conducted.

Methodology. This research involved a literature review of various articles related to the methods used in the WWW Accessibility Guidelines and issues related to cognitive disability. This research was conducted by the National Institute on Disability Research and Rehabilitation (NIDRR) in 2003 in terms of technology usage and cognitive disabilities and involved the development of accessibility evaluation tools and edification of the best practices for web access by disabled individuals. From this research, it was evident that the optimal method to achieve usability and accessibility of the internet by those with cognitive disabilities, has not yet been achieved. The role of this review was to ascertain the differences and similarities contained in the identified guidelines.

Three limitations were identified from the literature. These encompass the fact that cognitive elements have not been well defined as a learning disability. Therefore, this limitation makes it difficult to identify the right strategy to be used in web design as the various authors of the guideline noted that there were conflicting types of disabilities and cognitive disabilities within these guidelines. Further, the complex method of sharing information with persons with disabilities, has also not been succinctly defined. Secondly, there is a dearth of outcomes-oriented efficacy of guideline usability. Lastly, there is a possibility of that certain relevant guidelines may have been omitted from this review, despite extensive research having been conducted (Friedman \& Bryen, 2007).

Results, analysis and discussion. This analysis included cognitive disability and specific web design recommendations. Additionally, 20 websites were identified for this analysis. The disabilities considered incorporated cognitive impairment, cognitive disability, mental retardation, dyslexia and intellectual disability. Each guideline was examined for specific recommendations. About $15 \%$ of the design guidelines showed these disabilities to be important. Most website guidelines indicated that 22 of the respondents recommended that use of text size and shape, text writing styles, shape and size and navigation consistency and screen layout were critical elements to accommodate learning disabilities. As a consequence of this analysis, the web design recommendations are indicated in Table 1 below.

Conclusion. The internet has become part and parcel of everyday activity. People have come to rely on the WWW as a major medium for communication, information and commerce. However, it has brought about a large digital divide between those that can use the technology and those that cannot. In this case, cognitively disabled persons have been left behind in this digital progression. Thus, web accessibility guidelines have been introduced to reduce the effects of this imbalance. Design guidelines represent a standardized design approach for achieving equity in this field. Adoption of standardized web design recommendations should be encouraged to ensure that websites can suit a specific method of web design. In the guidelines, websites should use clear and simple texts. Use of WWW content accessibility guidelines such as 2.0 (Caldwell, Cooper, Reid, \& Vanderheiden, 2008) might serve as global standardized web accessibility guidelines or regulations across countries. Further research should be conducted to identify various types of barriers experienced by cognitive disability and how these can reduced. 
1. Use pictures, icons and symbols along with text.

2. Use clear and simple text.

3. Consistent navigation and design on every page.

4. Use headings, titles and prompts.

5. Support screen readers. Use alternate text tags.

6. Use larger fonts, fonts in minimum 12pt or $14 \mathrm{pt}$.

$30 \%$

7. Uncluttered, simple screen layout.

$30 \%$

8. Maintain white space: Use wide margins.

9. Website customizable, control of: type size, placement of navigation (right, left side) contrast, large print, sound.

10. Use exit, home, help, next page buttons on every page.

$\begin{array}{ll}\text { 11. Use with sans serif fonts, such as Arial, Verdana, Helvetica, Tahoma. } & 20 \%\end{array}$

$\begin{array}{lr}\text { 12. Navigation buttons clear, large, and consistent. } & 20 \%\end{array}$

$\begin{array}{ll}\text { 13. Use numbered lists rather than bullets. } & 20 \%\end{array}$

$\begin{array}{lr}\text { 14. Support font enlargement for Web browsers. } & 15 \%\end{array}$

$\begin{array}{lr}\text { 15. Use color for contrast. } & 15 \%\end{array}$

$\begin{array}{ll}\text { 16. Check reading level with automated tool. } & 15 \%\end{array}$

$\begin{array}{lc}\text { 17. Don't right justify text; use ragged edge right hand margins. } & 15 \%\end{array}$

$\begin{array}{lr}\text { 18. Use Lower case, no ALL CAPS. } & 15 \%\end{array}$

$\begin{array}{ll}\text { 19. Provide voice captions (audio files) for text. } & 15 \%\end{array}$

$\begin{array}{ll}\text { 20. Provide audio/voice-overs where the words are read aloud. } & 15 \%\end{array}$

21. Use navigation methods, i.e. 'undo' or 'back button' to help user recover when lost. $\quad 15 \%$

22. Give feedback on a user's actions (e.g. confirm correct choices, alert users to errors or possible errors). $\quad 15 \%$

Table 1: Web Design Recommendations (Borg, Berman-Bieler, \& Khasnabis, 2015).

\section{References}

Borg, J., Berman-Bieler, R., \& Khasnabis, C. (2015). Assistive technology for children with disabilities: Creating opportunities for education, inclusion and participation-a discussion paper. Geneva: $W H O$.

Caldwell, B., Cooper, M., Reid, L. G., \& Vanderheiden, G. (2008). Web content accessibility guidelines (WCAG) 2.0. W3C, 4-13.

Dobransky, K., \& Hargittai, E. (2016). Unrealized potential: Exploring the digital disability divide, Poetics, 58, $18-28$.

Friedman, m. G., \& Bryen, D. N. (2007). Web accessibility design recommendations for people with cognitive disabilities. Technology, 3-4.

HKSAR. (2008). Child assessment service. Department of Health, HKSAR. Retrieved from https://www.dhcas.gov.hk/. 